\title{
Photoselective Vaporization of the Prostate in Men with a History of Chronic Oral Anti-Coagulation
}

\author{
Omer F. Karatas, Erdal Alkan, Kaya Horasanli, Huseyin Luleci, Kemal Sarica
}

Department of Urology, Memorial Hospital (OFK, EA, HL, KS), and Department of Urology (KH), Sisli Etfal Medical Research and Training Hospital, Istanbul, Turkey

\begin{abstract}
Purpose: A considerable percentage of patients with benign prostatic hyperplasia (BPH) also have additional cardiac pathologies, which often require anticoagulant therapy. The aim of this study was to evaluate the efficacy and safety of photoselective vaporization of the prostate (PVP) for BPH in cardiac patients receiving anticoagulant therapy.

Materials and Methods: A total of 67 patients suffering from BPH and high risk cardiac pathologies were operated on using laser prostatectomy. All patients had cardiac pathologies with bleeding disorders requiring anticoagulant use, and underwent standard urologic evaluation for BPH. Patients were treated with laser prostatectomy for relief of the obstruction using the KTP/532 laser energy at $80 \mathrm{~W}$.

Results: The mean patient age was 71.4 years (range 55-80). Mean prostate volume on transrectal ultrasonography was $73.2 \mathrm{~mL}$ (range 44-120). Operation time ranged from 40 to $90 \mathrm{~min}$, with an average value of $55 \mathrm{~min}$. The average hospital stay was 48 hours (range 12-72) and the Foley catheters were removed within 48 hours, with a mean catheterization time of $34.2 \pm 5.9$ hours $(0-48)$. No patient required an additional procedure due to severe bleeding necessitating intervention during the early postoperative phase. Mean International symptoms scoring system (IPSS) values and post voiding residual volume decreased and peak urinary flow rate increased $(\mathrm{p}<0.001)$. Our results showed that the mean prostate volume had decreased by $53 \%$ at 6 months.

Conclusions: High-power photo selective laser vaporization prostatectomy is a feasible, safe, and effective alternative for the minimal invasive management of $\mathrm{BPH}$, particularly in cardiac patients receiving anticoagulant therapy.
\end{abstract}

Key words: prostate; prostatic hyperplasia; bladder outlet obstruction; laser surgery; anticoagulants Int Braz, J Urol. 2010; 36: 190-7

\section{INTRODUCTION}

Both medical and surgical options for the treatment of benign prostatic hyperplasia $(\mathrm{BPH})$ have expanded in recent years (1). Concerning the surgical treatment of this pathology, despite its effective and successful results, transurethral resection of the prostate (TURP) has been found to be associated with significant risks for the patient. While intraoperative mortality rates have been reported to be from $0 \%$ to $0.8 \%$, morbidity (especially bleeding and perforation) rates were $6.9 \%$ to $14 \%$ (range $2-5$ ). Morbidity in the month after surgery varied from $9.5 \%$ to $18 \%$ (range 2-5) and consisted mainly of bleeding with or without clot retention, which can necessitate re-operation or transfusion. Moreover, an increase in the risk of deep venous thrombosis of $6.8 \%$ to $10 \%$ in normal patients undergoing TURP has previously been reported (6). 
Regarding the management alternatives in patients receiving oral anticoagulant, studies have clearly demonstrated that instead of temporarily ceasing anticoagulant drugs (which may increase the risk of thromboembolic processes) physicians tended to stop anticoagulant agents for a certain period preoperatively and to use heparin intravenously during this phase (7).

Oral anticoagulant therapy is commonly used in atrial fibrillation, recurrent thromboembolic disorders, and prosthetic heart valves. TURP adds an additional risk in these patients with an additional BPH pathology (6). Therefore, an alternative surgical technique to TURP is needed.

Concerning the various potential therapeutic strategies for minimally invasive treatment of $\mathrm{BPH}$ that could be associated with less morbidity than TURP, various types of laser prostatectomy have been used in the last $10-15$ years $(8,9)$. Although several alternatives have been used to reduce the known perioperative morbidity, perioperative hemorrhage remains the major complication of this specific intervention (10). Previous studies have shown that visual ablation of the prostate with the neodymium : yttrium-aluminium-garnet (Nd: YAG) and holmium laser could be performed in patients under anticoagulant treatment with satisfactory hemostatic results (11-13). In particular, holmium laser enucleation of the prostate is a safe and effective therapeutic modality in patients on anticoagulation with symptomatic $\mathrm{BPH}$ refractory to medical therapy.

As regards this procedure, the $80 \mathrm{~W}$ KTP (potassium titanyl phosphate) laser vaporization of the

Table 1 - Indications for oral anticoagulant therapy.

\begin{tabular}{lc}
\hline Indications* & N. Pts \\
\hline Myocardial infarction & 36 \\
Prosthetic heart valves & 9 \\
Congestive heart failure & 3 \\
Pacemaker & 13 \\
Aortic aneurysm & 1 \\
Chronic atrial fibrillation & 21 \\
Total & 83 \\
\hline
\end{tabular}

* More than 1 pathology in some patients. prostate combines the tissue-debulking properties of transurethral resection of the prostate with the known good hemostatic properties of other laser techniques. It is a safe procedure for the patient and provides a virtually bloodless operation and immediate improvement of voiding (14-18).

In the present prospective clinical study we aimed to evaluate the efficacy of high-power photoselective laser vaporization prostatectomy for the minimal invasive management of BPH in cardiac patients receiving anticoagulant therapy.

\section{MATERIALS AND METHODS}

Between May 2004 and September 2005, a total of 67 patients (referred with complaints of infravesical obstruction and high risk cardiac pathologies) were retrospectively included in the study program. While the majority of the patients had had bypass surgery $(58 / 67,86.5 \%)$ due to ischemic heart disease, the remaining had valvular pathology and had undergone replacement surgery. Conservative management along with the use of alpha blockers had failed to alleviate the symptoms in these patients.

All patients underwent standard urologic evaluation for BPH with the International symptom scoring system (IPSS) symptom score, peak urinary flow rate (Qmax), ultrasound prostate volume estimation, prostate-specific antigen, and digital rectal examination. In addition, blood loss and serum sodium levels were determined preoperatively and on day 1 postoperatively in patients during intervention and observed in all patients. Patients included in the study program were on anticoagulant therapy for various indications (Table-1). Forty-six patients were on acetyl salicylic acid, 12 patients were on clopidrogel bisulfate, and 9 patients were on warfarin sodium medication.

Anticoagulant medication was stopped 2 days before the surgery in all patients. Subcutaneous Low molecular weight heparin (LMWH) was started 24 hours after the discontinuation of oral anticoagulant (warfarin sodium) in 9 patients due to the coagulation risk and stopped 12 hours before surgery when the International Normalized Ratio (INR) decreased to less than 1.4. LMWH was restarted 12 hours after 
surgery and oral warfarin sodium was resumed after the patients were able to receive medication ( 24 hours after the last LMWH application). After INR increases to greater than 2, LMWH can be discontinued. No significant complication occurred in any patient due to the cessation of the medication; in 9 patients, however, anticoagulant therapy was maintained using enoxaparin. Furthermore, the 58 patients on acetyl salicylic acid or clopidrogel bisulfate medication did not receive any anticoagulant therapy during the surgery and these agents were restarted 3 days after photoselective vaporization of the prostate surgery. All patients presented with an American Society of Anesthesiologists score of 2 or lower and, thus, were considered to be at low surgical risk.

All patients were treated with laser prostatectomy for relief of the obstruction. Indications for surgery were the same as previously described in detail (18). During the laser procedure, KTP/532 laser energy at $80 \mathrm{~W}$ was delivered by a $6 \mathrm{~F}$ side-firing fiber through a $24 \mathrm{~F}$ continuous-flow cystoscope (KTP/532 laser; Nigara PV trade mark laser system; Laserscope, San Jose, CA). PVP using saline irrigation was performed under spinal or general anesthesia. The prostatic lobes were readily vaporized to within capsular fibers. The mean duration ranged from 40 to 90 minutes with an average time of 55 minutes, during which a mean of $122.7 \pm 24 \mathrm{~kJ}$ (range 30-280) of energy was delivered. A urethral catheter was inserted postoperatively. On rare occasions, if hematuria persisted despite intermittent irrigation, continuous irrigation was begun using a 3-way catheter and bladder washouts required were recorded. The catheter was routinely removed within 48 hours of the operation. Details of adverse events were noted, including any pyrexia greater than 38 degrees $\mathrm{C}$, hypotension episodes (systolic blood pressure less than $100 \mathrm{mmHg}$ ), and blood transfusion or clot retention episodes. When patients were able to void adequately, they were discharged from the hospital. Postoperative efficacy parameters were mean and percentage changes from baseline in hemoglobin, sodium level (12 hours after the operation), IPSS, peak urinary flow rate (Qmax), post-void residual urine volume, and transrectal ultrasound prostate volume measurement.

The patients were reassessed at 3 and 6 months postoperatively with respect to any changes in these parameters. The Mann-Whitney U and Friedman test was used to assess statistical significance.

\section{RESULTS}

A total of 67 patients on anticoagulation were included in this study, of whom 54 (81\%) presented with symptomatic BPH refractory to medical treatment. Table-2 lists the main characteristics of all patients. Before surgery, mean preoperative INR was $1.32(0.9-2.5)$ in those receiving warfarin treatment. These values for the patients on warfarin anticoagulation and LMWH substitution were $2 \pm 0.22$ and 1.20 \pm 0.15 , respectively. Applied energy (kJ) was $226 \pm$ 56 and we used only one fiber for each patient.

No patient required an additional procedure due to severe bleeding necessitating intervention during the early postoperative phase and no blood transfusion was required before, during, or after the procedure. In 5 patients $(7.4 \%)$ receiving LMWH treatment, however, prolonged bleeding (mean 2.4 days) was observed and continuous bladder irrigation and washout were necessary in these patients. Two other patients receiving LMWH (2.9\%) were referred with bleeding 7-10 days after the procedure, which was treated conservatively. Thus, a total of 7 patients $(10.4 \%)$ experienced bleeding after laser surgery and were subsequently treated appropriately with no major consequences. On the other hand, in $5(55.5 \%, 5 / 9)$ patients receiving LMWH, prolonged bleeding was observed. Similarly, of the 2 patients with delayed bleeding this represented $2 / 9$ (22.2\%) of those on LMWH. Therefore, $7 / 9$ patients $(77.7 \%)$ on LMWH experienced bleeding, which represented $10.4 \%$ of patients in the entire series. Immediately after the procedure, 8 patients $(11.9 \%)$ experienced 1 to 7 days of mild irritative symptoms requiring no specific treatment, and 5 patients $(7.4 \%)$ had urgency for 10-14 days but none had incontinence, newly developed impotence, or required re-operation. Five patients $(7.4 \%)$ required re-catheterization for about 3 days due to transient urinary retention following the removal of the urethral catheter. Mean preoperative and postoperative hemoglobin was $12.6 \mathrm{~g} / \mathrm{dL}$ (range 8.9 to 14.8 ) and $11.7 \mathrm{~g} / \mathrm{dL}$ (range 8.0 to 13.2 ), respectively $(\mathrm{p}<0.62)$. Mean preoperative and postoperative 
Table 2 - Baseline characteristics and preoperative parameters in patients who underwent photoselective vaporization of the prostate surgery.

\begin{tabular}{lc}
\hline & Mean (range) \\
\hline Age (years) & $71.4 \pm 9(55-80)$ \\
Prostate vol. (cc) & $73.2 \pm 33(44-120)$ \\
Surgery indications (\%): & \\
$\quad$ LUTS refractory to medical treatment & $54(81 \%)$ \\
$\quad$ Urinary retention & $12(18 \%)$ \\
$\quad$ Hematuria & $1(1 \%)$ \\
Qmax (mL/sec) & $6.8 \pm 2.9$ \\
Residual urine (mL) & $123.6 \pm 108.6$ \\
IPSS & $24.3 \pm 5.9$ \\
PSA level (ng/mL) & $4.52 \pm 3.7$ \\
\hline
\end{tabular}

$I P S S=$ International symptom scoring system $;$ LUTS = lower urinary tract symptoms; Qmax $=$ maximum flow.

serum sodium was 138.7 (range 131 to 145 ) and 138.0 (range 128 to 142 ), respectively $(\mathrm{p}<0.82)$. Evaluation of the pre- and postoperative hemoglobin and plasma sodium levels did not show any significant difference. Despite the significant change with respect to hemoglobin values after the procedure, this change was not clinically significant. Saline solution $(0.9 \%)$ was used for irrigation intraoperatively and fluid absorption was not observed in any of the patients.

The perioperative and postoperative data of those who underwent LMWH and those that did not are presented (Table-3). Of the sexually active patients $62 \%$ had retrograde ejaculation at 3 and 6 months. No other significant complications were observed. Again no patient required admission to the intensive care unit for severe cardiac problems (such as myocardial infarction) during the study.

The voiding parameters showed improvement during the early phase of follow-up. The mean prostate volume had decreased by $53 \%$ after 6 months (Table-4).

At the end of the 6 months, no postoperative complications developed in any patients, i.e. urinary tract infection, stress incontinence, cloth retention, bladder neck contracture, or urethral stricture.

\section{COMMENTS}

$\mathrm{BPH}$ is the most common cause of bladder outlet obstruction in the elderly and it is the most frequent pathology requiring surgical treatment in men. Concerning the treatment of this condition, medical and surgical options for the treatment infravesical obstruction have expanded in recent years (1). Among the surgical techniques, the gold standard treatment, TURP, has been applied with significant success rates for years and it significantly improves urinary symptoms and urinary flow. However, it is well known that, despite its common acceptance and widespread application, complications can be seen in up to $20 \%$ of cases following a successful intervention (10,19-21). Currently, there are a number of minimally invasive procedures that may be safe and effective alternatives to TURP. Among these, one promising surgical technique is laser prostatectomy $(8,9,22)$.

Over the past $10-15$ years a variety of endoscopic laser techniques have evolved for the treatment of BPH that have been associated with less morbidity than TURP with a shorter hospital stay. The initial type of laser in this field, the Nd: YAG laser, has allowed the performance of prostatectomy in an almost 
Table 3 -Perioperative and postoperative outcomes and follow-up results (LMWH (+) the patients who had low molecular weight heparin before the PVP surgery and LMWH (-) those who did not.)

\begin{tabular}{lccc}
\hline & LMWH $(+)$ & LMWH $(-)$ & p Value* \\
\hline $\begin{array}{l}\text { No. of patients } \\
\text { Mean perioperative } \pm \text { SD }\end{array}$ & $9(13 \%)$ & $58(87 \%)$ & \\
$\quad$ Resection time (min) & $52.5 \pm 14.8$ & $48.7 \pm 12.6$ & 0.07 \\
$\quad$ Serum hemoglobin change (gm/dL) & $-0.4 \pm 0.6$ & $-0.3 \pm 0.5$ & 0.72 \\
$\quad$ \% Serum hematocrit change & $-0.4 \pm 1.1$ & $-0.5 \pm 2.1$ & 0.66 \\
$\quad$ Serum sodium change (mmol/dL) & $-0.5 \pm 1.8$ & $-0.2 \pm 2.2$ & 0.81 \\
Postoperative & $5(7 \%)$ & & \\
$\quad$ No. bladder washouts & $1.9 \pm 1.2$ & $1.8 \pm 1.3$ & 0.01 \\
$\quad$ Mean postoperative stay \pm SD (days) & $2.2 \pm 1.4$ & $2.1 \pm 1.7$ & 0.83 \\
$\quad$ Mean catheterization time \pm SD (days) & & & \\
No. of complications & $4(6 \%)$ & $1(1 \%)$ & 0.01 \\
$\quad$ No. pts with prolonged bleeding & $2(3 \%)$ & - & - \\
$\quad$ Re-hospitalization for secondary hemorrhage & $5(7 \%)$ & 0 & 0.01 \\
$\quad$ Re-hospitalization for acute urinary retention & & & \\
\hline
\end{tabular}

* Mann Whitney U test; PVP = photoselective vaporization of the prostate; LMWH = Low molecular weight heparin; PSA $=$ prostatespecific antigen; Qmax = maximum flow; $S D=$ standard deviation.

bloodless field and without absorption of irrigant, but long-term follow-up data have demonstrated that sloughing of the remaining necrotic tissue may cause bladder outlet obstruction and related symptoms for a definite period of time after treatment. Moreover, patients undergoing these types of laser treatment required a longer period for maximum improvement, which probably reflects the lack of tissue debunking at the time of surgery.
Another type of laser used for obstruction relief in BPH patients is the holmium laser application and this type of laser has been reported to be an effective alternative to the "gold standard" for large prostates, entailing significantly less blood loss and a much shorter catheter time and hospital stay $(1,2,15)$.

Several studies of high risk cases with different laser types have been performed in the past. In

Table 4 - Comparative evaluation of all parameters before and after photoselective KTP laser prostatectomy.

\begin{tabular}{lcccc}
\hline Parameter & Before treatment & 3 months & 6 months & p Value* \\
\hline Q-max (mL/sec) & $6.8 \pm 2.9$ & $21.5 \pm 8.8$ & $24.5 \pm 9.6$ & $<0.001$ \\
IPSS symptom score & $24.3 \pm 5.9$ & $8.2 \pm 2.3$ & $7.9 \pm 2.6$ & $<0.001$ \\
Mean prostate vol. $(\mathrm{mL})$ & 73.2 & $29.7(-59 \%)$ & $24.8(-53 \%)$ & $<0.01$ \\
Residual urine $(\mathrm{mL})$ & $123.6 \pm 108.6$ & $43.1 \pm 32.9$ & $26.2 \pm 18.9$ & $<0.001$ \\
Mean PSA level $(\mathrm{ng} / \mathrm{mL})$ & 4.52 & 4.18 & 4.64 & $<0.01$ \\
\hline
\end{tabular}

* Friedman test $;$ KTP = potassium titanyl phosphate; IPSS = International symptom scoring system. 
contrast to laser types previously used, the potassium titanyl phosphate (KTP) laser has been shown to vaporize prostatic tissue with minimal coagulation of the underlying structures. With use of the KTP laser, heat has been found to be concentrated into a small volume, which causes the ablation of the tissue by rapid vaporization of cellular water by leaving a 2$\mathrm{mm}$ rim of coagulated tissue. After favorable results were obtained in studies of animal models and human cadavers, the clinical use of 60-80 W KTP laser prostatectomy began in selected patients $(4,6,7,9,10)$. Again ex vivo studies have demonstrated that larger coagulation zones during $80 \mathrm{~W}$ KTP laser vaporization make this technique a relatively bloodless ablative procedure, giving rise to hemostasis, that is highly superior to conventional TURP-like tissue resection (17). Decreased morbidity (bleeding or other cardiac high risk disorders) and shorter hospital stay have been reported as the factors resulting in the rapid acceptance of photoselective vaporization of the prostatic tissue.

Yuan et al. have reported 12 months' results from a prospective clinical trial in 128 high-risk men with benign prostatic hyperplasia who underwent photoselective vaporization of the prostate (23). According to their results, the mean operation time was $51.6(22.8) \mathrm{min}$, the mean catheterization time was 2.8 (1.6) days, IPSS decreased from 19.2 (6.1) before surgery to 6.1 (4.6), Q(max) increased from 7.0 (2.8) $\mathrm{mL} / \mathrm{s}$ to $24.8(8.0) \mathrm{mL} / \mathrm{s}$, and residual urine decreased from $168(89) \mathrm{mL}$ to $23(34) \mathrm{mL}, 12$ months later. All these results were similar to our clinical outcomes.

On the other hand, Ruszat et al. and Woo et al. reported that there was no perioperative discontinuation in drug administration since withdrawal would have posed a considerable risk for thromboembolic events $(24,25)$. We stopped anticoagulant medication 2 days before surgery in all patients but started LMWH, 24 hours after the discontinuation of oral anticoagulant because of the coagulation risk. We did not observe any complications linked to the discontinuation of anticoagulant treatment after the surgery.

New-generation, high-power potassium titanyl phosphate lasers can be used in the day-care setting, with minimal risk of complications and without the need for postoperative catheter drainage $(10,11,17)$.
Furthermore, the ability to use this technology in the management of relatively large prostates, in a safe and effective manner, represents another advantage of this technique that makes it preferable to the other surgical alternatives including open prostatectomy or TURP.

Based on our relatively short follow-up data, we suggest that high-power KTP lasers represent a very significant challenge to the 'gold standard' status of TURP. KTP laser prostatectomy has been found to be sufficiently effective in our patients receiving anticoagulant therapy due to certain cardiac problems. Successful results similar to those produced by TURP including significant improvements both in IPSS symptom scores as well as maximum flow rates were observed in the majority of patients. Again residual urine volume decreased significantly after laser vaporization. Limited complications were observed and apart from the mild dysuria and urgency no major complication that really affects the quality of life in the treated patients was observed in our series. The urethral catheter was removed within $48 \mathrm{~h}$ in all patients and most of the patients were discharged the following day. More importantly, although mild hematuria lasting a couple of days after the procedure was observed in some patients, severe hemorrhage requiring intervention was not observed in any patient.

Thus, our data confirmed the hemostatic efficacy of KTP laser vaporization during its TURPlike resection with highly effective tissue removal especially in high-risk patients by giving rise to hemostasis. On the other hand, 7/9 patients on LMWH experienced minor bleeding complications in our study and so this subgroup had a particular risk although none required intervention or transfusion. Despite all these successful results, we must emphasize that the relatively small number of patients and short follow-up period, the absence of a control group (TURP group), and the small number of patients who used LMWH during the procedure could be considered the drawbacks of our study.

\section{CONCLUSION}

Our results demonstrate that high-power photoselective laser vaporization prostatectomy is feasible 
and appears to be safe and effective for quickly relieving bladder outlet obstruction due to BPH in a day-care setting with minimal risk of complications and minimal need for postoperative catheterization. Although several factors limit the interpretation of the results of this study, the procedure could be considered as a promising alternative in the treatment of BPH in all but especially in high-risk patients receiving anticoagulant therapy. However, we suggest that larger randomized clinical trials to compare this technique with standard TURP and long-term follow-up data are needed to determine its efficacy, safety, and durability.

\section{CONFLICT OF INTEREST}

None declared.

\section{REFERENCES}

1. Lam JS, Cooper KL, Kaplan SA: Changing aspects in the evaluation and treatment of patients with benign prostatic hyperplasia. Med Clin North Am. 2004; 88: 281-308.

2. Horninger W, Unterlechner H, Strasser H, Bartsch G: Transurethral prostatectomy: mortality and morbidity. Prostate. 1996; 28: 195-200.

3. Mebust WK, Holtgrewe HL, Cockett AT, Peters PC: Transurethral prostatectomy: immediate and postoperative complications. A cooperative study of 13 participating institutions evaluating 3,885 patients. J Urol. 1989; 141: 243-7.

4. Uchida T, Ohori M, Soh S, Sato T, Iwamura M, Ao T, et al.: Factors influencing morbidity in patients undergoing transurethral resection of the prostate. Urology. 1999; 53: 98-105.

5. Doll HA, Black NA, McPherson K, Flood AB, Williams GB, Smith JC: Mortality, morbidity and complications following transurethral resection of the prostate for benign prostatic hypertrophy. J Urol. 1992; 147: 1566-73.

6. Hedlund PO: Postoperative venous thrombosis in benign prostatic disease. A study of 316 patients, using the 125I-fibrinogen uptake test. Scand J Urol Nephrol. 1975; (27 suppl): 1-100.

7. Tscholl R, Straub W, Zingg E: Electroresection of the prostate in patients treated with heparin. J Urol. 1980; 124: $221-2$.
8. Te AE: The development of laser prostatectomy. BJU Int. 2004; 93: 262-5.

9. Aho TF, Gilling PJ: Laser therapy for benign prostatic hyperplasia: a review of recent developments. Curr Opin Urol. 2003; 13: 39-44.

10. Fitzpatrick JM, Mebust KW: Minimally Invasive and Endoscopic Management of Benign Prostatic Hyperplasia. In: Walsh CW, Retik AB, Vaughan ED, Wein AJ (eds.), Campbell's Urology, 8 th edition. Philadelphia: WB Saunders. 2004; pp. 1402.

11. van Melick HH, van Venrooij GE, Boon TA: Laser prostatectomy in patients on anticoagulant therapy or with bleeding disorders. J Urol. 2003; 170: 18515.

12. Kabalin JN, Gill HS: Urolase laser prostatectomy in patients on warfarin anticoagulation: a safe treatment alternative for bladder outlet obstruction. Urology. 1993; 42: 738-40.

13. Le Duc A, Gilling PJ: Holmium laser resection of the prostate. Eur Urol. 1999; 35: 155-60.

14. Te AE, Malloy TR, Stein BS, Ulchaker JC, Nseyo UO, Hai MA, et al.: Photoselective vaporization of the prostate for the treatment of benign prostatic hyperplasia: 12-month results from the first United States multicenter prospective trial. J Urol. 2004; 172: 1404-8.

15. Barber NJ, Muir GH: High-power KTP laser prostatectomy: the new challenge to transurethral resection of the prostate. Curr Opin Urol. 2004; 14: 21-5.

16. Malek RS, Kuntzman RS, Barrett DM: High power potassium-titanyl-phosphate laser vaporization prostatectomy. J Urol. 2000; 163: 1730-3.

17. Sandhu JS, Ng CK, Gonzalez RR, Kaplan SA, Te AE: Photoselective laser vaporization prostatectomy in men receiving anticoagulants. J Endourol. 2005; 19: 1196-8.

18. Bachmann A, Ruszat R, Wyler S, Reich O, Seifert HH, Müller A, et al.: Photoselective vaporization of the prostate: the basel experience after 108 procedures. Eur Urol. 2005; 47: 798-804.

19. Westenberg A, Gilling P, Kennett K, Frampton C, Fraundorfer M: Holmium laser resection of the prostate versus transurethral resection of the prostate: results of a randomized trial with 4-year minimum long-term followup. J Urol. 2004; 172: 616-9.

20. Gürdal M, Tekin A, Yücebas E, Sengör F: Nd:YAG laser ablation plus transurethral resection for large prostates in high-risk patients. Urology. 2003; 62: 914-7.

21. Shingleton WB, Farabaugh P, May W: Three-year follow-up of laser prostatectomy versus transurethral 
resection of the prostate in men with benign prostatic hyperplasia. Urology. 2002; 60: 305-8.

22. Carter A, Sells H, Speakman M, Ewings P, MacDonagh $\mathrm{R}$, O’Boyle P: A prospective randomized controlled trial of hybrid laser treatment or transurethral resection of the prostate, with a 1-year follow-up. BJU Int. 1999; 83: 254-9.

23. Yuan J, Wang H, Wu G, Liu H, Zhang Y, Yang L: High-power $(80 \mathrm{~W})$ potassium titanyl phosphate laser prostatectomy in 128 high-risk patients. Postgrad Med J. 2008; 84: 46-9.
24. Ruszat R, Seitz M, Wyler SF, Abe C, Rieken M, Reich $\mathrm{O}$, et al.: GreenLight laser vaporization of the prostate: single-center experience and long-term results after 500 procedures. Eur Urol. 2008; 54: 893-901.

25. Woo H, Reich O, Bachmann A, Choi B, Collins E, de la Rosete J, et al.: Outcome of greenlight HPS 120-W laser therapy in specifi $\mathrm{c}$ patient populations: those in retention, on anticoagulants and with large prostates (> 80mL). Eur Urol Suppl 2008; 7: 378-83.

\section{Correspondence address:}

Dr. Omer Faruk Karatas

Department of Urology, Memorial Hospital

Piyale Pasa Bulvari, Okmeydani

34385, Istanbul, Turkey

Fax: + 90212 314-6621

E-mail: dr.omerfaruk@gmail.com 